\title{
Understanding the covalent inhibition of Clavulanate against $\beta$-lactamases (TEM-1 and KPC-2) with QM/MM screening methods.
}

\author{
Rubén Fritz $^{1 *}$, Jans Alzate-Morales ${ }^{1}$, Marc Van der Kamp², Jim Spencer ${ }^{3}$ and Adrian Mulholland² \\ 'Doctorado en Ciencias Aplicadas, Centro de Bioinformática y Simulación Molecular (CBSM), Facultad de Ingeniería, Universidad de Talca, Talca, Chile. \\ ${ }^{2}$ Centre for Computational Chemistry, University of Bristol, Bristol, UK. \\ ${ }^{3}$ Department of Molecular Bioscience, School of Cellular and Molecular Medicine, University of Bristol, Bristol, UK.
} *rfritz@utalca.cl

\section{Abstract}

$\beta$-lactamases are a primary cause of bacterial resistance to $\beta$-lactam antibiotics for many important human pathogens (particularly Gram-negative bacteria) [1] Inhibitors of $\beta$-lactamase have been implemented as a dual therapy with antibiotics, but there are only four inhibitors clinically approved and resistance to these compounds is also rising [2]. For $\beta$-lactam inhibitors, after acylation, the opening of five-membered ring leads to the formation of a transient imine intermediate then it rearranges several times to form a trans or cis final enamine inhibition-products. Slow hydrolysis of this product by the enzyme leads to an inhibited $\beta$-lactamase [2]. $A$ computational study of reaction mechanism for the first step on the deacylation of the inhibitor clavulanate with TEM-1 (inhibited) and KPC-2 (hydrolyzed) enzymes using QM/MM Umbrella Sampling with DFTB method is presented [3]. 2D free energies surfaces for the reactions were calculated using the weighted histogram analysis method (WHAM) and the minimum energy path (MEP) was identified; where the highest point along the MEP is taken as the transition state giving the activation free energy " $\Delta \mathrm{G}_{\text {calc }}$ ". Our results show that TEM-1 and $\mathrm{KPC}-2$ have an approximate $5 \mathrm{kcal} / \mathrm{mol}$ difference in $\Delta \mathrm{G}_{\text {calc. Such results are in good agreement with }}$ inhibition experimental data for two enzymes in which KPC-2 is less inhibited by clavulanate than TEM-1. We hope our methodology can assist the design and development of covalent inhibitors through a computational screening of inhibitory activity of other molecules.

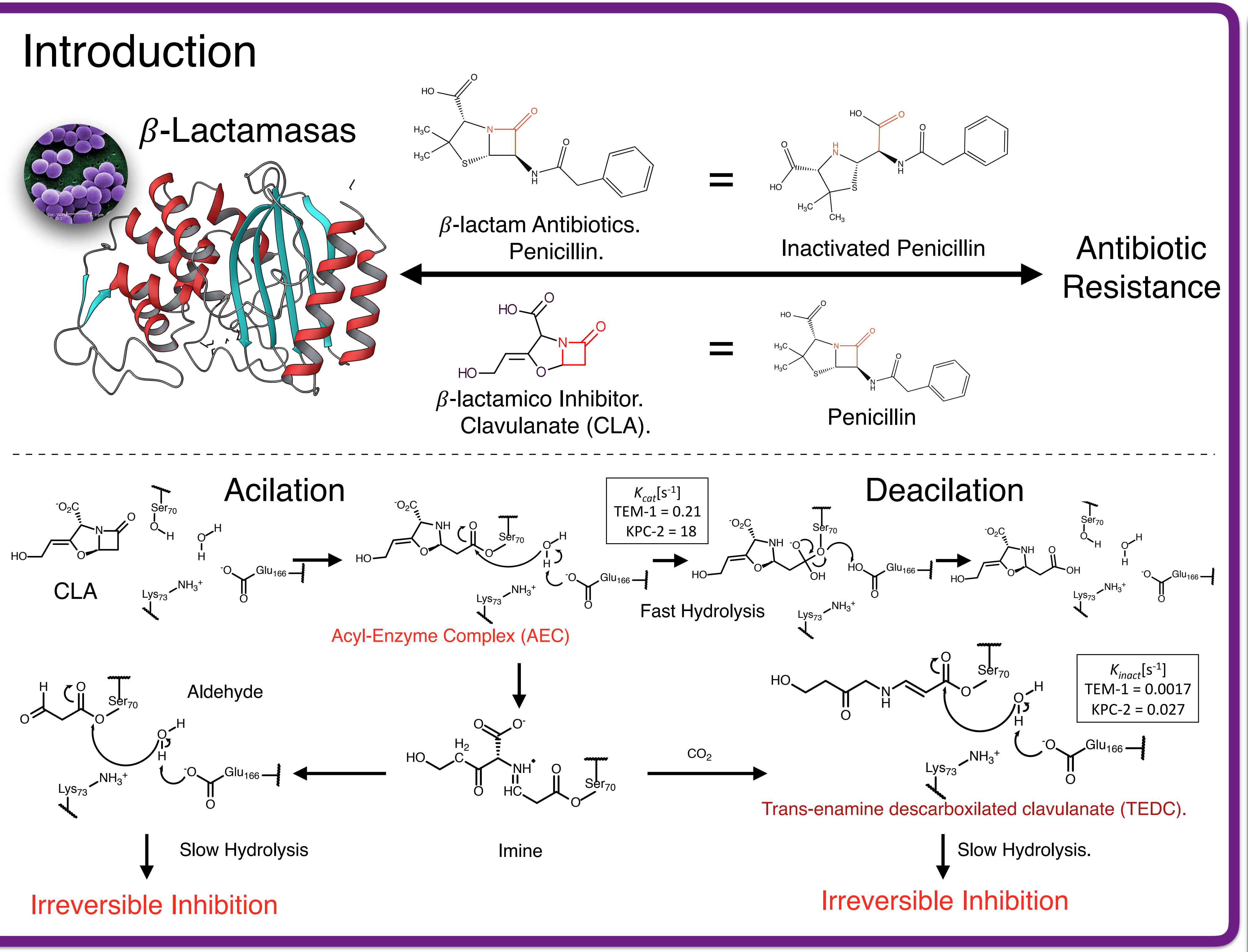

\section{Methodology}

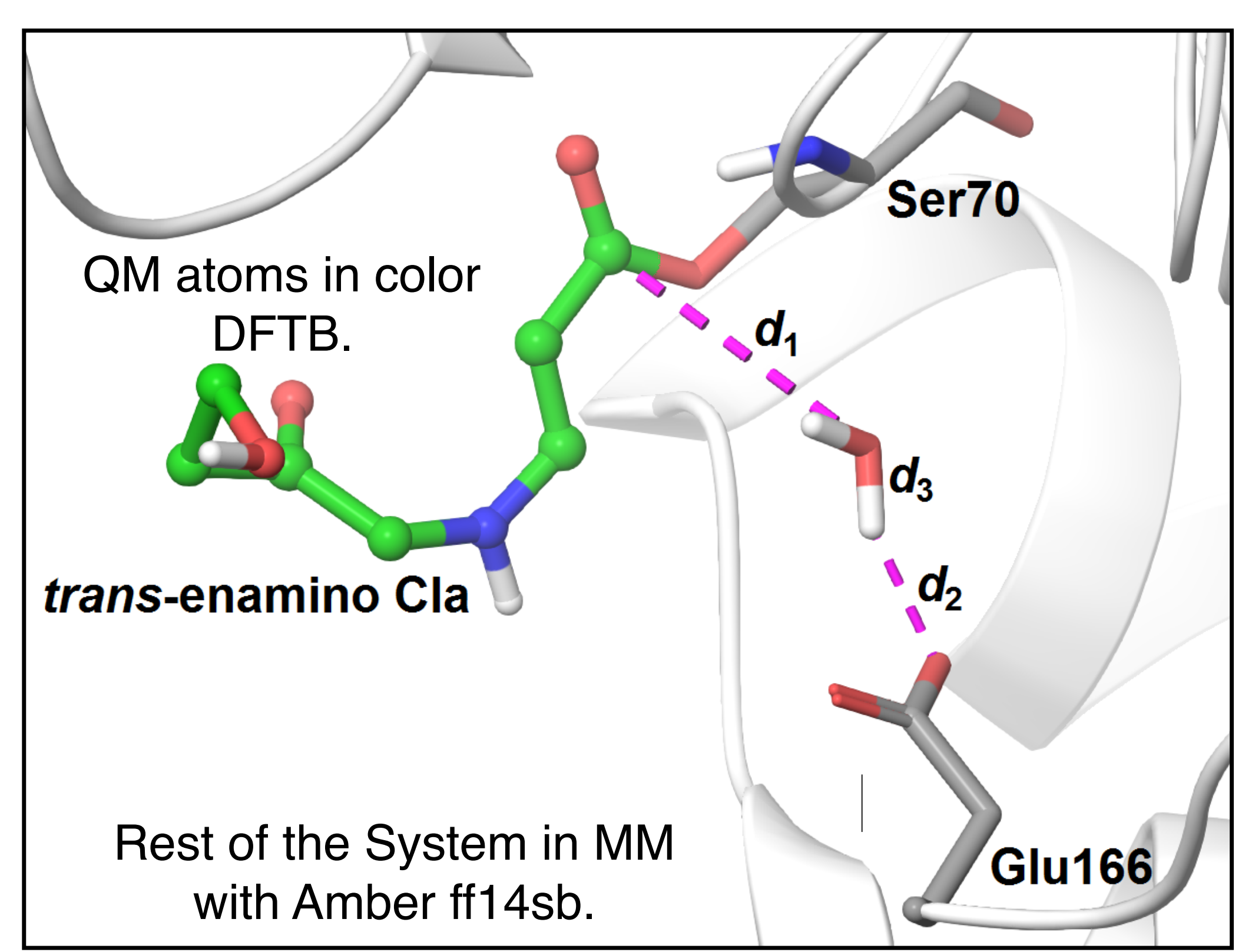

QM/MM MD Umbrella Sampling.

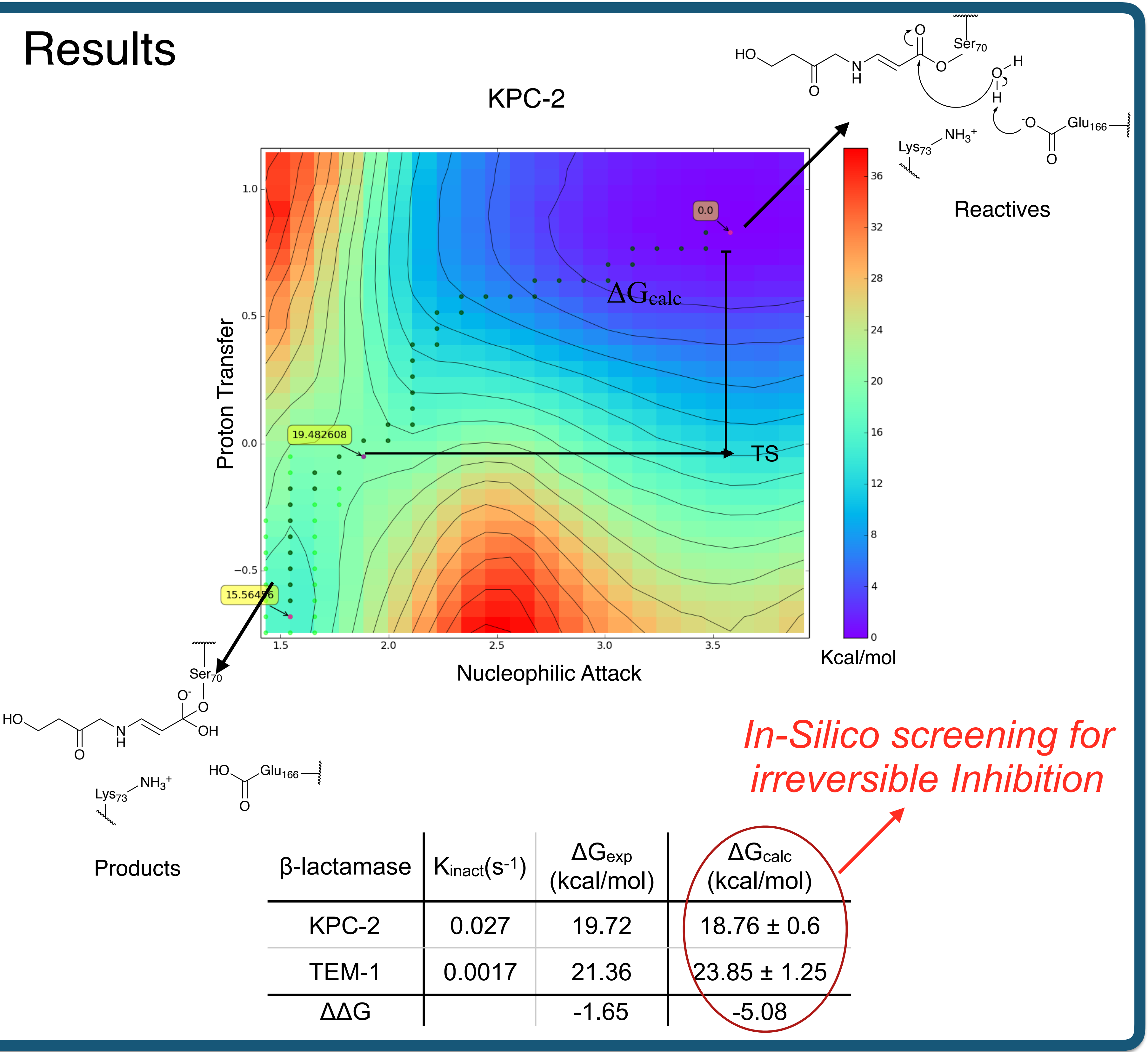

Conclusiones.

The activation free energies calculated for TEDC with TEM-1 and KPC-2 are in the same magnitud order as the experimental values for the inactivation constant.

These computational methodology aloud us to discriminate the inhibitory activity of CLA against KPC-2 and TEM-1 $\beta$-lactamase.

Perspectives.

Calculate others covalent complexes during CLA inhibition.

These protocol could potentially be use as a In-silico screening for inhibitory activity of covalent inhibitors.

- Expansion to others protein-ligands systems of medical interest.

Acknowledgments.

R.F. thanks The Royal Society of Chemistry (RSC) for economic support through Researcher Mobility Grant (R. Fritz 16 Round 1). J.A-M. and R.F. thanks financial support from project FONDECYT No. 1140618.

\section{References:}

[1] Hermann, J. C., Pradon, J., Harvey, J. N., \& Mulholland, A. J. J. Phys. Chem. A (2009), 113(43), 11984-11994 [2] Drawz, S. M., Papp-Wallace, K. M., \& Bonomo, R. a. Antimicrob. Agents Chemother (2014), 58(4), 1835-1846. [3] Chudyk, E. I., Limb, M. a L., Jones, C., Spencer, J., van der Kamp, M. W., \& Mulholland, A. J. Chem Commun, (2014), 50, 14736-14739. 